\title{
SISTEMA DE INTERACCIÓN FAMILIAR ASOCIADO A LA AUTOESTIMA DE MENORES EN SITUACIÓN DE ABANDONO MORAL O PROSTITUCIÓN
}

\begin{abstract}
Alaírdes María Ferreíra Rocha'
Estudio descriptivo sobre 160 menores: 80 menores se encontraban en situación de abandono moral o prostitución, y 80 menores con similares características, pero que no ejercían la prostitución. A quienes se les aplicó dos inventarios: El de adaptabilidad y cohesión familiar (FACES III) y el de autoestima de Coopersmith

Los resultados muestran que existen diferencias significativas entre las puntuaciones medias alcanzadas en los niveles de cohesión, adaptabilidad y autoestima entre el grupo de menores en situación de abandono moral que ejercía la prostitución y el grupo control.

Además se ha encontrado, que existe una alta asociación entre los tipos de familia (Balanceada, rango media y extrema) y los niveles de autoestima con el grupo de menores que se encuentran en situación de abandono moral o prostitución y aquellos que no se encuentran en esta situación. Existe, también, asociación entre niveles de autoestima en grupo de menores que se encuentran en situación de abandono moral con otro que no se encuentra.
\end{abstract}

PALABRAS CLAVES: Tipos de familia, adaptabilidad y cohesión familiar, autoestima, jóvenes en abandono moral y prostitución

This is a descriptive study about 160 youngsters from Lima: 80 that are involved in moral abandonment or prostitution and 80 of similar characteristics but not involved in prostitution. They were applied two inventories: Family adaptability and cohesion evaluation (FACES - III) and Self-esteem of Coopersmith.

The results show significative statistics differences between the means in cohesion, family adaptability and Self-esteem when the groups of youngsters inmoral abandonment and prostitution were compared with the control group.

Besides, there is a high relationship between the types of family (Balance, mean rank and extreme) and the levels of self-esteem with the groups of youngsters in maral danger or prostitution and those who are not in this condition.

KEY WORDS: Types of family, Family adaptability and cohesion and Self-esteem youngsters in moral abandonment and prostitution.

\footnotetext{
${ }^{1}$ Magister en Psicología Clínica. Dra. en Psicología. Miembro del CETADE (Centro de Estudia en alcohol y drogas de UFU)
} 


\section{INTRODUCCIÓN}

El tema de la prostitución es sumamente complejo) al hablar de este tópico es importante aclarar, en primer lugar, que forma parte de la industria de la comercialización del sexo. Entre las distintas modalidades de esta industria mencionamos la prostitución, el turismo sexual, el tráfico de niñas y la pornografía. Es conveniente esclarecer la magnitud de esta industria y también su globalización. Está presente en todos los países del mundo, pues responde a una cultura universal que ensalza la sexualidad masculina. La prostitución como la pornografía está fundamentada en la ideología patriarcal que confiere al hombre el derecho de adquirir el acceso al cuerpo de la mujer mediante la conquista, la violación o el pago de dinero, como sucede en la prostitución.

En Julio de 1999, el UNICEF declaró que la violencia contra las mujeres y los niños es «la más persuasiva violación de los derechos humanos en el mundo de hoy». Está estimado que 100 millones de niños a través del mundo viven en las calles; 40 millones de estos, en Latinoamérica. Muchos de estos niños están envueltos en actividades como mendigar, robar, o aun más, en la prostitución para sobrevivir.

Según documento de la OPS (1999), la prostitución de menores tiene que enfocarse de forma distinta a la de los adultos, pues entraña con mayor intensidad el uso del dominio y el poder, la coacción y explotación, la victimización y subordinación, la violencia y el abuso emocional, físico y sexual. La actividad sexual de menores a cambio de favores materiales involucra presión y amenazas. La prostitución de menores es una actividad ilegal y clandestina que merece sanciones judiciales. Sin embargo, la prostitución de menores crece y se hace cada vez más compleja en su articulación con otros sectores de la economía.

Los niños que han sido prostituidos se mantendrán con frecuencia envueltos en actividades criminales tales como robo o se envolverán en tipos de comportamiento antisocial. Muchos niños se convertirán en abusadores de químicos como una manera de sobrevivir a esta clase de vida. Se entregarán al uso de drogas, alcohol u otros solventes, como una forma de entumecerse contra el dolor que están sintiendo. En Latinoamérica, la mayoría de estos niños viven en las calles y tratan con crueldad severa sus propias vidas inhalando goma de zapatos. Aquí en Lima, particularmente, es común la inhalación en bolsa plástica de un pegamento denominado Terokal. Para las víctimas, la violencia, la desconfianza, la vergüenza y el rechazo pueden convertirse en norma y los niños pueden depender de sus explotadores en cuanto a estabilidad emocional y apoyo.

En la región de las Américas, el problema de la prostitución de menores aumentó durante los años ochenta, pero en los países ricos se manifestó de manera distinta que en los pobres. En todos ellos la característica más común era que el menor había sido objeto de negligencia y abuso emocional, físico y sexual en su propio hogar antes de prostituirse (Sereny, 1997). Sin embargo, los estudios indican que en los países en desarrollo la prostitución estaba relacionada principalmente con la necesidad de sobrevivir materialmente frente a la creciente pobreza y miseria que afectan a los países latinoamericanos.

Save the Children, ONG de Suecia, publicó en Lima, Perú (2002), unos Documentos de Trabajo sobre la prostitución de niñas, niños y adolescentes. En ellos se refiere que dentro de los varios factores condicionantes para el ingreso a la prostitución están: a) La precariedad de la familia, o sea, la prostitución se ve facilitada por la fragilidad del vínculo familiar. Cada vez más la familia es externa a sí misma. 
Muchos padres y madres son visitantes en sus propias casas. Su relación con los hijos se torna absolutamente frágil. La precariedad de las relaciones al interior de la familia, el abandono, el maltrato, el abuso, la violación y, en general, la carencia afectiva -como apuntan los Documentos- producen traumas muy fuertes en la autoestima. Los niños explotados sexualmente se sienten inútiles y pierden el valor ante sí mismos. La depresión seguida del suicidio es común en la vida de estos menores. Para superar el dolor de estas heridas muchos de ellos buscan en las drogas un camino para el escape, pues ya no soportan más los sentimientos de minusvalía, humillación y de pérdida de dignidad; b) La fragilidad de la escuela es otro factor condicionante de la prostitución, señalan los Documentos de Trabajo. Según el estudio, cada año un promedio de 125 mil niños abandonan la educación secundaria. En los últimos cuatro años medio millón de adolescentes han abandonado los estudios; la gran mayoría va a pararse en las esquinas, incorporándose a las bandas, la delincuencia, la microcomercialización de drogas y la prostitución. Los Documentos señalan que las escuelas no cuentan con programas para familias y los pocos como las escuelas de padres no cumplen su función, pues están impregnados de una catequesis acerca de lo que los padres deben hacer con los hijos en el marco de una superficialidad que ahuyenta en lugar de motivar y atraer; c) Los clientes son otro factor condicionante. En una encuesta aplicada en los bares y discotecas del puerto del Callao (Lima, Perú) se encontró que un requisito para trabajar en estos lugares era ser menor de 18 años. Cumplida esta edad, lo que queda es la calle o los prostíbulos. Los clientes son generalmente tripulantes de barcos, que pagan más, y en dólares, a cambio de ciertas exigencias; d) Factores condicionantes para las situaciones de la calle. La presencia de muchos niños en la calle se explica por situaciones de violencia familiar impregnadas de altos niveles de ignorancia y desconocimiento acerca de la responsabilidad que les corresponde a los padres en la educación de sus hijos y sobre los derechos que los asisten.

Las condiciones que favorecen la extensión del problema de los niños de la calle son la violencia familiar y la desestructuración de la familia debido a la ausencia del padre $\mathrm{O}$ la madre, o cuando éstos son alcohólicos, o el propio padre es un abusador sexual, o la madre tiene otras parejas que son una amenaza permanente.

El UNICEF (2001) en Ginebra, publicó un informe sobre la explotación sexual comercial de la infancia. El informe describe minuciosamente el alcance y las consecuencias del problema e incluye testimonios de mujeres, niños y niñas que han sido objeto de este tipo de abuso. Según el documento, las causas subyacentes de la explotación sexual comercial de la infancia incluyen la pobreza, la discriminación en materia de género, la guerra, el crimen organizado, la mundialización, los problemas en las familias y el comercio de las drogas.

El documento de trabajo realizado por el UNICEF recomienda, entre otros factores, el fortalecimiento y el restablecimiento de los vínculos familiares, o la creación de nuevos lazos afectivos para la convivencia familiar y comunitaria y la recuperación de la integridad física, psicológica y emocional de las víctimas.

También recomienda la reconstrucción de la propia imagen, la autoestima y la dignidad, el desarrollo de habilidades que permitan a los niños, niñas y adolescentes actuar con mayor seguridad ante posibles situaciones de riesgo.

El UNICEF, señala que entre $20 \%$ y $50 \%$ de las prostitutas en Lituania son menores de edad. Niñas de sólo 11 años trabajan en 
prostíbulos y niños de sólo 10 años son alojados en hogares infantiles, que han sido utilizados para rodar películas pornográficas.

El mismo estudio señala que en los Estados Unidos uno de cada cinco niños y niñas que ingresan en la línea de la Internet reciben requerimientos por parte de extraños para entablar relaciones sexuales. En otro estudio realizado en los Estados Unidos se reveló que cada año hay unos 104.000 niños y niñas víctimas de abuso sexual.

Según Bellamy (2001), Directora Ejecutiva del UNICEF, el abuso sexual de los niños y las niñas transforma completamente su mundo del revés. Debido a que puede provenir de un pariente cercano o de un amigo, el abuso sexual convierte en enemigos a las mismas personas a quienes los niños se dirigen para obtener protección: aquellos a quienes conocen y aman, y en quienes confían. Y debido a que estos hechos se pueden producir en los lugares donde viven, aprenden y juegan, transforman estos entornos familiares, como el hogar o la escuela, en espacios prohibidos y peligrosos.

El Movimiento El Pozo, el Fondo de Población de las Naciones Unidas y el INEI (1998), realizaron una investigación sobre la prostitución en el Perú, cuyo resultado señala que la Policía Nacional del Perú detuvo a 14 personas por proxenetismo. En el mismo año hubo 36 procesos judiciales por proxenetismo y 56 fueron procesados por proxenetismo. El resultado final muestra que en todo el año se emitieron 7 sentencias judiciales, 4 absolutorias y 3 condenatorias. Esto resulta en que todo el esfuerzo del sistema de justicia penal frente al problema del proxenetismo se reduce a apenas 3 sentencias judiciales condenatorias, una de las cuales fue una condena condicional y la otra se suspendió. Es decir, sólo una tuvo sanción efectiva.

En un estudio realizado por el UNICEF (1995) en Caracas, de las observaciones se establece que: de cada 100 menores afectados por la prostitución, 40 están en la miseria, 40 en pobreza y 20 tienen sus necesidades básicas satisfechas. Este estudio confirma la hipótesis de que hay alta correlación entre pobreza y prostitución de menores afectados con necesidades básicas no satisfechas.

Otros trabajos realizados por la Cámara de Comercio de Bogotá (UNICEF, 1991), muestra que la prostitución infantil es un tipo de respuesta a la necesidad del trabajo para subsistir, causado por la presión de la miseria, por el subdesarrollo económico social y cultural, por el desequilibrio de la distribución de los ingresos y la desprotección del sector marginal.

En Chile, Frez (1992), observa que los menores que ejercen la prostitución se encuentran situados en los niveles más bajos de la estructura social.

Brasil, después de Tailandia, tiene la segunda cantidad más alta de niños en prostitución en el mundo. UNICEF (1998) reporta esta estadística de aproximadamente 500.000. Probablemente no es una coincidencia el que Brasil también posea el tercer número más alto de casos de SIDA.

Según declaró Bellamy, C. (UNICEF, 2001), a millones de niños en todo el mundo se les compra y se les vende como si fuera objetos y se les utiliza como esclavos sexuales. Se trata de una violación completamente intolerable de los derechos de la infancia. En los Estados Unidos también se ha visto un aumento en prostitutas juveniles trabajando en las calles. Aunque esto es aún principalmente un fenómeno de las áreas urbanas más grandes tales como Los Ángeles y Nueva York, está moviéndose dentro de más ciudades medianas tales como Minneapolis y Seattle. Canadá ha experimentado una tendencia similar, en la cual no es fuera de lo común encontrar prostitutas menores de edad trabajando en las calles 
de Vancouver y Montreal.

Se constata a través de estudios realizados sobre la prostitución de menores, que la mayoría de ellos tuvieron sus primeras experiencias sexuales con personas de la propia familia. La mala comunicación familiar, las necesidades emocionales no satisfechas y el rol no protector de los padres podrían contribuir a los riesgos de abuso sexual en el menor.

El daño emocional también no puede ser subestimado. Los síntomas psicológicos experimentados por el menor en la prostitución, como la baja autoestima y depresión, son usualmente severas. Los períodos de ataques de depresión son comunes entre los niños prostituidos. Esto hace que el tomar decisiones sea dificultoso. Estos niños están totalmente inmovilizados por su depresión. Esto puede dar como resultado común el suicidio (UNICEF, 1998).

Por otro lado, son escasos los estudios efectuados en Perú sobre la prostitución infantil, la mayoría se aboca al estudio sobre el abandono moral en general. Así Cárdenas (1989), en su estudio empírico «Autoestima en niños urbano-marginales y su relación con el nivel de soporte social brindado por padres y pares» resalta la importancia y necesidad del soporte de los padres y de los pares en la autoestima de los niños de las zonas urbanomarginales de Lima Metropolitana.

En un trabajo sobre la autoestima y el abandono moral en un contexto social, Achancaray y cols. (1999) concluyen que la asertividad, el autorrespeto, la autodisciplina y la complementariedad son valores que los niños deben poseer en todo momento, ya que de ellos depende su inserción en la sociedad de una manera eficaz y con una seguridad de sí mismos, Castillo (1999), analizó la autoestima y su relación con la percepción de las actitudes de los progenitores en niños de condición socioeconómica media y baja», señala que la autoestima de estos menores está íntimamente relacionada con las actitudes de los padres; en ese sentido, recomienda que los progenitores deben tomar conciencia sobre la importancia de este valor para que sus hijos puedan enfrentar los diversos problemas cotidianos que se presentan y se presentarán en el futuro.

Un informe preparado por la ONG Acción por los Niños (1998) afirma que el cien por ciento de las niñas prostitutas, especialmente en Lima, no considera lo que hace como prostitución sino como un trabajo más.

En el Perú, la problemática de la prostitución en niños no difiere mucho de los otros países de Latinoamérica. En Lima, según un documento del UNICEF (1998), los menores se encuentran trabajando en la prostitución en zonas como el puerto del Callao, las playas, hoteles, y otros centros comerciales. No existe investigaciones o cifras para la prostitución de menores en Lima, ya que la prostitución está incluida en los registros policiales bajo el encabezamiento general de «Abandono y Riesgo Moral». Sin embargo, en una redada policial de niños en las calles de Lima en abril de 1984, 51 mujeres menores fueron recogidas en varios lugares conocidos y detenidas por prostitución.

\section{Teoría de Olson}

La evaluación de la percepción que los menores tienen de sus respectivos sistemas familiares se basa en el Modelo Circunflejo de David H. Olson. El modelo plantea tres dimensiones centrales del comportamiento familiar: Cohesión, Adaptabilidad y Comunicación. Estas dimensiones permite establecer 16 categorías de familias, las cuales según el grado de funcionalidad de su interacción se ubican en tres rangos: familias balanceadas, las cuales podrían establecer un equilibrio en los niveles de apego y de flexibilidad para el cambio, gracias a su adecuado proceso de comunicación; familias de 
rango medio, las cuales serían extremas en alguna de las dimensiones de cohesión o adaptabilidad, pero balanceadas en la otra; y la familia extremas, las cuales tendrían muy altos o muy bajos niveles de apego y de reflexibilidad.

La cohesión se define como el vínculo emocional que los miembros de la familia tienen entre sí. La cohesión familiar evalúa el grado en que los miembros de la familia están separados o conectados a ella. Dentro del Modelo Circunflejo, los conceptos específicos para medir y diagnosticar la dimensión de cohesión familiar son: vinculación emocional, límites, coaliciones, tiempo, espacio, amigos, toma de decisiones, interés y recreaciones.

La adaptabilidad familiar tiene que ver con la medida en que el sistema familiar es flexible y capaz de cambiar. Se define como la habilidad de un sistema marital o familiar para cambiar su estructura de poder, las relaciones de roles y las reglas de las relaciones, en respuesta al estrés situacional y propio del desarrollo. Los conceptos específicos para diagnosticar y medir la dimensión de adaptabilidad es: poder en la familia (asertividad, control, disciplina), estilo de negociación, relaciones de roles y reglas de las relaciones.

La comunicación familiar es la tercera dimensión y facilita el movimiento en las dimensiones de la cohesión y adaptabilidad. La comunicación familiar es un proceso interactivo en el que la comunicación es siempre una acción conjunta. Para la análisis, interesa más la forma y el contexto en que la comunicación actúa. Las habilidades de comunicación positiva, tales como empatía,

reflexiva y comentarios de apoyo, permiten las familias compartir entre si sus necesidades y preferencias cambiantes en relación con la cohesión y la adaptabilidad. Las habilidades negativas tales como doble mensajes, dobles vínculos y críticas, disminuyen la habilidad para compartir los sentimientos y restringen por lo tanto la movilidad de la familia en las otras dimensiones.

Olson (1985), considera que la interacción de la dimensión de cohesión y adaptabilidad condiciona el funcionamiento familiar. Cada una de estas dimensiones tienen 4 rasgos y su correlación determina 16 tipos familiares, integradas en tres niveles de funcionamiento familiar. Se describe algunos conceptos que permitirán una mejor interpretación:

\section{Cohesión}

La dimensión de cohesión, es considerada unificante y tiene dos componentes: el vínculo emocio.nal de los miembros de la familia y el grado de autonomía indivi4ual que una persona experimenta en la familia. (Gráfico 1).

La cohesión determina 4 niveles:

Dispersa:

- Extrema separación emocional.

- Falta de lealtad familiar.

- Se da muy poco involucramiento o interacción entre sus miembros.

- La correspondencia afectiva es infrecuente entre sus miembros.

- Hay falta de cercanía parento-filial.

- Predomina la separación personal.

- Rara vez pasan el tiempo juntos.

- Necesidad y preferencia por espacios separados.

- Se toma las decisiones independientemente.

- El interés se focaliza fuera de la familia.

- Los amigos personales son vistos a solas. 
- Existen intereses desiguales.

- La recreación se lleva a cabo individualmente.

\section{Conectada}

- Hay cercanía emocional.

- La lealtad familiar es esperada.

- Se enfatiza el involucramiento pero se permite la distancia personal.

- Las interacciones afectivas son alentadas y preferidas.

- Los límites entre los subsistemas son claros con cercanía parento- filial.

- La necesidad de separación es respetada pero poco valorada.

- El tiempo que se pasa junto es importante.

- El espacio privado es respetado.

- Se prefieren las decisiones conjuntas.

- El interés se focaliza dentro de la familia.

- Los amigos individuales se comparten con la familia.

- Se prefieren los intereses comunes.

- Se prefieren la recreación compartida, que la individual.

\section{Aglutinada:}

- Cercanía emocional extrema.

- Se demanda lealtad hacia la familia.

- El involucramiento, el altamente simbiótico.

- Los miembros de la familia dependen mucho unos de otros.

- Se expresa la dependencia afectiva.

- Hay extrema reactividad emocional.

- Se dan coaliciones parento-filiales.

- Hay falta de límites generacionales.

- Hay falta de separación personal.

- La mayor parte del tiempo se pasa junto.

- Se permite poco tiempo y espacio privado.

- Las decisiones están sujetas al deseo del grupo.

- El interés se focaliza dentro de la familia.

- Se prefieren los amigos de la familia a los personales.

- Los intereses conjuntos de dan por mandato.

\section{Separada:}

- Hay separación emocional.

- La lealtad familiar es ocasional.

- El involucramiento se acepta

- prefiriéndose la distancia personal.

- Algunas veces se demuestra la correspondencia afectiva.

- Los límites parento-filiales son claros con cierta cercanía entre padres e hijos.

- Se alienta cierta separación personal.

- El tiempo individual es importante pero se pasa parte del tiempo, juntos. 





Se prefieren los espacios separados compartiendo el espacio familiar.

- Las decisiones se toman individualmente siendo posibles las decisiones conjuntas.

- El interés se focaliza fuera de la familia.

- Los amigos personales raramente son compartidos con la familia.

- Los intereses son distintos.

- La recreación se lleva a cabo más separada que en forma compartida.

\section{Adaptabilidad}

La dimensión de adaptabilidad es la habilidad de un sistema marital o familiar para cambiar su estructura de poder, sus roles y reglas de relación en respuesta a una demanda situacional o de desarrollo. Para que exista un buen sistema de adaptación se requiere un balance entre cambios y estabilidad. El desplazamiento entre alta y baja adaptabilidad determina 4 tipos:

\section{Caótica:}

- Liderazgo limitado y/o ineficaz.

- Las disciplinas son muy poco severas habiendo inconsistencia en sus consecuencias.

- Las decisiones paren tales son impulsivas. Hay falta de claridad en las funciones. Existe alternancia e inversión en los mismos.

- Frecuentes cambios en las reglas que se hacen cumplir inconsistentemente.

\section{Estructurada:}

- En principio el liderazgo es autoritario, siendo algunas veces igualitario.

- La disciplina rara vez es severa, siendo predecible sus consecuencias.

- Es un tanto democrática.

- Los padres toman las decisiones.

- Las funciones son estables pero pueden compartirse.

- Las reglas se hacen cumplir firmemente. Pocas son las que se cambian.

\section{Rígida:}

- El liderazgo es autoritario, existiendo fuerte control parental.

- La disciplina es estricta, rígida y su aplicación severa.

- Es autocrática.

- Los padres imponen las decisiones.

- Los roles están estrictamente definidos.

- Las reglas se hacen cumplir estrictamente, no existiendo la posibilidad de cambio.

\section{Flexible:}

- El liderazgo es igualitario, permite cambios.

- La disciplina es algo severa negociándose sus consecuencias.

- Usualmente es democrática.

- Hay acuerdo en las decisiones.

- Se comparten los roles o funciones.

- Las reglas se hacen cumplir con flexibilidad.

- Algunas reglas cambian. 


\section{Niveles de funcionamiento familiar}

La correlación entre las dimensiones de cohesión y adaptabilidad, determina 3 niveles de funcionamiento familiar. En el rango balanceado se ubican las familias de óptimo funcionamiento, mientras que en el extremo se ubican las familias no funcionales.

\section{Tipo Balanceada}

Las familias de esta categoría son centrales en ambas dimensiones, y se encuentran al centro del círculo. Hablamos de las familias: flexiblemente separada, flexiblemente conectada, estructuralmente separada, estructural mente conectada.

En los sistemas abiertos, los individuos se distinguen por su habilidad por experimentar y balancear los extremos de independencia y dependencia familiar. Tienen la libertad de estar solos o conectados al miembro de la familia que elija. El funcionamiento es dinámico por lo que pueden cambiar. La familia es libre para moverse en la dirección que la situación, que el ciclo de vida familiar o la socialización de un miembro de la familia lo requiera. Ese tipo de familia es considerado el más adecuado.

\section{De rango medio}

Las familias de esta categoría son extremas en una sola dimensión. Hablamos de las familias: flexiblemente dispersa, flexiblemente aglutinada, caóticamente separada, caóticamente conectada, estructuralmente dispersa, estructuralmente aglutinada, rígidamente separada, rígidamente conectada.

El funcionamiento de estas familias presenta algunas dificultades en una sola dimensión, las cuales pueden ser originadas por momentos de estrés.

\section{Extremas}

Las familias de esta categoría son extremas tanto en las dimensiones de cohesión como adaptabilidad. Hablamos de la familia: caóticamente dispersa, caóticamente aglutinada, rígidamente dispersa, rígidamente aglutinada.

Tiene un funcionamiento menos adecuado. Olson considera varios aspectos al respecto:

Las familias extremas son una forma exagerada de las de rasgo medio, ya que pertenecen a un continuo de características que varían en intensidad, cualidad y cantidad.

Que, en «momentos» especiales de la vida familiar, un funcionamiento extremo puede ser benéfico para la estabilidad de los miembros.

En muchos casos, el problema de un miembro de la familia o pareja pueden hacerlo considerar el funcionamiento familiar como extremo; por ejemplo: si un miembro de la pareja desea el divorcio considerará que la familia tiene funcionamiento extremo.

Los grupos extremos (Olson, 1982, 1983) pueden funcionar bien por el tiempo que «todos» los miembros de la familia lo deseen así. Esto es importante porque hay grupos culturales que determinan funcionamientos familiares extremos.

\section{Enfoque teórica sobre la autoestima}

Coopersmith (1967), psicólogo que más ha trabajado en esta área, concluyó que las personas basaban la imagen de sí mismo en los siguientes criterios:

a) Significación: El grado en que sienten los sujetos que son amados y aceptados por 
aquellos que son importantes para ellos.

b) Competencia: Capacidad para desarrollar tareas que consideran importantes.

Virtud: Consecución de valores morales y éticos.

Poder: Grado en que pueden influir en su vida y en los demás.

La evaluación de Coopersmith (1959) de los padres con hijos de alta autoestima, sugiere la importancia de tres condiciones generales en el hogar: Primero, los padres comunican en forma clara su aceptación del niño y el niño percibe que ellos pertenecen la familia, que son miembros valiosos; segundo, los padres comunican límites bien definidos y alta expectativa para su ejecución. El niño reconoce las expectativas de sus padres sobre su comportamiento y perciben la seguridad de sus padres en sus habilidades; y tercero, los padres respetan la individualidad del niño, permitiéndole ser diferentes y únicos sin imponerles límites generales establecidos. Contrarios a otros escritores, Coopersmith encuentra que la alta autoestima y la creatividad son más probables que provengan de ambientes bien estructurados, con límites y exigencias que de ambientes abiertos. El sugiere que una demanda apropiada para alcanzar mayor competencia crea capacidad improvisada, que el niño reconocerá a su propio crecimiento y que tomarán un sentido más fuerte de competencia y valor en sus logros.

El afán de competencia y el nivel de autoestima de los padres son también factores importantes. El niño observa la seguridad de sus progenitores, su aproximación frente a situaciones que implican desafío y su método para tratar con dificultades o disgustos. El niño puede obsérvalos evaluar tanto sus puntos positivos como sus deficiencias cuando se enfrenta a las críticas. Las expectativas positivas del niño son más probables de ser ejecutadas si es que ha observado a sus padres modelar esos atributos.

Un supuesto eslabón entre la inseguridad del individuo y las prácticas de los padres es lo que Coopersmith llama «poder en las relaciones». Los niños sienten su propio poder porque sus padres respetan sus opiniones y derechos, reconocen la individualidad de sus hijos, dándoles grandes oportunidades para que escojan su propia dirección, crean confianza del niño, imbuyéndolo a resistir la presión para conformarse sin su propia aprobación.

A pesar de que se conoce relativamente poco acerca de la autoestima, generalmente se considera que es un atributo personal altamente favorable. Se han escrito diversos libros y artículos, dedicados a la higiene mental y desarrollo de la personalidad, que refieren que la autoestima es uno de los elementos primordiales que ejerce uno de los más altos grados de influencia en el funcionamiento de la personalidad del ser humano Rodríguez, (1976) Y Branden, (1987).

Muchos de los enfoques tradicionales para el entendimiento de los orígenes de la autoestima tienden a enfatizar el aprendizaje social e interpersonal. Básicamente estas opiniones sugieren, que los individuos adquieren gradualmente creencias, acerca de ellos mismos que son un reflejo del modo en que ellos son tratados por su ambiente social (Bandura, 1987). En esencia las personas tienden a verse y valorizarse del mismo modo en que ellos son vistos y valorizados por otros. Por supuesto hay excepciones a esta orientación general.

\section{Influencia de los padres}

Para toda persona, son los padres los que juegan un papel preponderante en nuestras vidas, los que ejercen mayor influencia y los que hacen vemos como personas capaces o incapaces, valorables, invalorables, sensibles, insensibles, etc.

Y en el caso de percibirse como sujetos incapaces, ineficaces, desamparados, la mayoría 
tratará de buscar la aceptación y/o aprobación de estos, presentándose como una necesidad, la cual seguiremos buscando aún después que estos hayan fallecido.

Tal vez muchas veces no se logra conseguir su aprobación y no queda más que darse a uno mismo la aceptación, tratando de trasmitirla a los hijos, lo que les va a proporcionar un escudo psicológico, como menciona Mc Kay (1991), lo cual los protegerá a lo largo de la vida.

El ayudar a los hijos a desarrollar una adecuada autoestima es una tarea primordial para todos los padres que desean ver a sus hijos libres de serios conflictos psicológicos, así como también lejos del alcohol, drogas, delincuencia, etc.

Así estos serán capaces de llevar una vida sana, donde puedan salir adelante, tomando riesgos, experimentado situaciones nuevas y fortaleciendo aún más su autoestima.

Para los hijos pequeños, los padres son todo, son tomados como modelos, adquiriendo así una serie de conductas y/o carácterísticas. Los niños que no son atendidos, escuchados, a los cuales no se les dedica cierta cantidad de tiempo, se percibirán como personas menos importantes, carentes de valor, donde sus necesidades no tienen valor alguno. Adquiriendo así desde temprana edad una baja autoestima.

A medida que van creciendo ya no son los padres, las figuras principales sino que ahora también lo son los amigos, maestros y los encargados de cuidarlos, pero sin embargo, toman los valores básicos que le dieron sus primeros modelos.

Muchos de los alcances de sentido común para desarrollar la autoestima, están basados en la expectación de que el niño gradualmente internaliza creencias de ellos mismos, los cuales les son comunicados por medio de su ambiente social. La tarea de la paternidad es particularmente compleja porque los niños son inicialmente incapaces de responder a un conjunto interno de expectaciones. Los padres no pueden enseñarles simplemente a enfrentar y confrontar salidas como ellos mismos lo hacen, porque el balance del niño entre el control de emociones y el control de pensamientos es muy distinto. Creando un sentido general de sentimientos propios positivos, requieren una estrategia protectora, una visión global que tome en cuenta la inmadurez desarrollada; y planear actividades a los padres para que cambien estratégicamente a medida que el niño madura.

Existe la creencia según la teoría del amor incondicional de que los niños con alta autoestima son aquellas que han sido criados con tanto amor y elogio que están de algún modo, libres de fallas, y protegidos de humillaciones. Esta teoría del amor incondicional para construir autoestima, algunas veces, usa una metáfora: «el balde de amor» si los padres mantienen este balde de amor, lleno al tope, nada puede ir mal, las decepciones deben ser suavizadas rápidamente, como si fueran inyecciones peligrosas. Los padres deben trabajar extra para construir la realidad, así el niño nunca cuestionará el yo o sus habilidades. Ellos por lo tanto, deben competir contra el posible ego y las confusiones fuera de la familia. El error, por supuesto es que los problemas complejas especialmente humanos, son raramente resueltos simplemente con prodigar más elogios o más amor o más dinero en ellos. Esto es, en su núcleo, una decisión defensiva, evasiva que teme enfrentarse a lo que puede ser para el niño un defecto realmente pequeño y nutre más bien una baja autoestima.

Coopersmith (1967) define a la autoestima como la evaluación que todo individuo hace y mantiene con respecto a sí mismo, expresando una actitud de aprobación o desaprobación indicando que tan capaz, significativo, exitoso y digno se encuentra o siente. La autoestima de una persona es un juicio de mérito que se expresa mediante las actitudes que mantiene hacia el ego. Es una experiencia subjetiva transmitida a otros mediante reportes orales y otros comportamientos expresivos.

Considerando a la autoestima así como la autoevaluación global tanto positiva como negativa que toda persona hace con respecto a sí misma, permaneciendo estable de una situación a otra e inclusive durante años. 
Cabe resaltar que Coopersmith hizo énfasis, también en la importancia de la experiencia familiar en el desarrollo de la autoestima, especialmente durante los primeros años de vida, donde los individuos adquieren la imagen de sí mismo, la cual más tarde les servirá de base para su futura auto evaluación y por tanto para su autoestima.

\section{PROBLEMA}

Con base a lo expuesto, surge la necesidad de realizar un estudio que indague básicamente: ¿En qué medida difieren la interacción familiar y la autoestima, al compararse con un grupo de menores que se encuentra en situación de abandono moral, con otro que no presenta esta condición?

Así mismo se estudia la asociación que existe entre la percepción del tipo de familia y los niveles de autoestima que tienen los menores en situación de abandono moral y de aquellos que no se encuentra en dicha situación.

\section{HIPÓTESIS}

H1: Existen diferencias significativas entre las puntuaciones medias alcanzadas en cohesión, adaptabilidad y autoestima al compararse a un grupo de menores en situación de abandono moral con otro grupo que no está en esta condición.

H2: Existe asociación entre los niveles de autoestima en un grupo de menores que se encuentra en situación de abandono moral con otro grupo que no se encuentra en esta condición.

H3: Existe relación entre la percepción del tipo de familia y la autoestima que tienen los menores en situación de abandono moral.

H4: Existe relación entre la percepción del tipo de familia y la autoestima que tienen un grupo de menores que no se encuentra en situación de abandono moral.

\section{MÉTODO}

Este trabajo tiene un carácter descriptivo - comparativo, habiéndose seleccionado dos muestra: una con menores en situación de abandono moral o prostitución, y otra con menores que no se encuentran en tales condiciones se procedió a igualar ambos grupos de acuerdo a su edad cronológica, grado de escolaridad, nivel socioeconómico con fines de contrastación.

Por otro lado, la investigación es correlacional porque se orienta a la determinación del grado de relación existente entre dos o más variables de interés: autoestima y tipo de familia en una misma muestra de sujetos.

La población está constituida por menores víctimas de abandono moral o prostitución, y proceden de las Comisarías de los Distritos de La Victoria, San Juan de Lurigancho y del Cercado de Lima y también de los clubes nocturnos y calles del Cercado de Lima que son cubiertas por la ONG INPPARES (Instituto peruano de Paternidad Responsable). Así como por menores que están en situación de riesgo pero que no ejercen la prostitución.

El método de muestreo es no probabilística (no aleatoria) de tipo intencional porque se basa exclusivamente en la selección de los casos más convenientes para el propósito del estudio.

La muestra inicial estuvo constituida por 293 y la muestra final una vez depurada quedó conformada por 160 niñas (80 niñas en situación de abandono moral y 80 niños sin abandono moral) que se presenta en la tabla $\mathrm{N}^{\circ} 1$ : 


\section{Tabla 1: Muestra efectiva con y sin situación de abandono en población de riesgo $(\mathrm{N}=80)$}

\begin{tabular}{|c|c|c|c|}
\hline EDAD & Sin abandono & En abandono mora & Ambos \\
\hline 13 & 6 & 6 & 12 \\
\hline 14 & 11 & 11 & 22 \\
\hline 15 & 16 & 16 & 32 \\
\hline 16 & 17 & 17 & 34 \\
\hline 17 & 30 & 30 & 60 \\
\hline TOTAL & 80 & 80 & 160 \\
\hline
\end{tabular}

\section{Las Variables de estudio fueron:}

Niveles de cohesión Niveles de adaptabilidad

Niveles de autoestima

Abandono moral con o sin prostitución

\section{Técnicas e instrumentos de recolección de datos}

Ficha de datos demográficos

Cuestionario Faces III David Olson

Cuestionario de Autoestima Coopersmith

\section{Cuestionario FACES III de Olson}

Desarrollado para evaluar las dos dimensiones mayores del Modelo Circunflejo, como son cohesión y adaptabilidad familiar.

El Modelo permite que una persona clasifique a su familia en 16 tipos específicos o en tres grupos más generales, que corresponden a familias. Balanceadas, de rango medio y familias extremas.

La prueba consta de 20 ítems: 10 ítems corresponde a cohesión y 10 ítems a adaptabilidad. Los resultados relativos a la dimensión de cohesión se distribuyen en 2 ítems para cada una de los siguientes aspectos: lazos emocionales (11-19), límites familiares (7-5), intereses comunes y recreación (13-15), coaliciones (1-17), tiempo y amigos (9-3).

La dimensión adaptabilidad se distribuye en dos ítem para cada uno de los siguientes aspectos: Liderazgo (6-18), control (122), Y disciplina (4-10), y, 4 ítem para roles y reglas de relación (8-14-16-20).

Respecto a la validez del FACES III, se redujo la correlación entre cohesión y adaptabilidad a casi cero, resultando en dos dimensiones claramente independientes. La adaptación realizada en el Perú fue realizada por Reusche (1994), a través del sistema de jueces y de un análisis cualitativo.

La confiabilidad se determinó mediante el coeficiente Alpha de Cronbach para cada una de las dimensiones: cohesión y adaptabilidad, datos que se presentan en la tabla 2 . La confiabilidad, determinada por Test-retest, con intervalos de 4 a 5 semanas, arrojó para cohesión: $\mathrm{R}=0.83$; y para adaptabilidad, $\mathrm{R}=0.80$.

La adaptación realizada en el Perú fue aplicada por Reusche (1994), a través del sistema de jueces y de un análisis cualitativo.

\section{Inventario de Autoestima}

El inventario de Autoestima, Stanley Coopersmith (1959) se encuentra constituido por 50 ítems, referidos a las autopercepciones del individuo con respecto a las siguientes áreas: Padres, Pares, el Sí mismo y el Colegio. 
La descripción hecha en 1984 por Prewitt-Díaz, es muy explícita; y define al instrumento como un Inventario de Autoreporte de 58 ítems, en el cual el sujeto lee una sentencia declaratoria para decidir, si ésta es «igual que yo»o «distinto a mí».

El Inventario está referido a la percepción del estudiante en cuatro áreas: Autoestima General, Autoestima Social, Hogar y Padres, Escolar-Académica y encontrándose también una Escala de mentira compuesta por 8 ítems.

El área de autoestima general $(G)$ está referida a la evaluación general que presenta el sujeto con respecto a sí mismo, dando a conocer el grado en que éste se siente seguro, capaz, valioso, exitoso y significativo.

El área de autoestima social $(S)$ evalúa tanto la participación como el liderazgo del sujeto frente a las diversas actividades con jóvenes de su misma edad, como también el que se pretende como un sujeto abierto y firme al dar sus puntos de vista, dándolos a conocer sin limitación alguna.

El área escolar $(E)$ evalúa el grado de satisfacción del individuo frente a su trabajo escolar y Ia importancia que le otorga, así como también el interés de ser interrogado ido cuestionado en el salón de clase.

El área hogar-padres $(H)$ mide el grado en que el sujeto se siente aceptado por su entorno familiar. Es decir, en que medida es comprendido, amado respectado y considerado por cada uno de los miembros de su familia.

Escala Mentira $(M)$. Si en ésta área el puntaje bruto resulta ser superior a 10, significaría que las respuestas del sujeto son poco confiables e invalidan la aplicación del inventario.

El estudio de la validez del inventario de autoestima se efectuó mediante el análisis de las correlaciones con la prueba de personalidad de California que mide Adaptación Personal y Social. Se desarrolló una matriz de correlaciones entre el inventario de Coopersmithy la prueba de California y la mayor parte de los coeficientes hallados alcanzaron valores significativos y los valores bajos fueron las correlaciones de la escala de mentira de Coopersmith con las escalas de California.

El coeficiente alfa de confiabilidad para el Inventario de Autoestima fue de 0.84 para la versión en español utilizada en Puerto Rico (Prewitt-DÍaz, 1979). En la estandarización realizada en Chile fue similar: 0.87 y 0.88 .

Para el análisis estadístico de los datos se usó el programa SPSS, versión 10.

\section{RESULTADOS}

\section{Estudio de la confiabilidad}

Para analizar la confiabilidad de las pruebas de Cohesión, Adaptabilidad y Autoestima hemos hecho uso el análisis de consistencia interna alfa de Cronbach.

Los alfa encontrados para las pruebas (Cohesión, Confiabilidad y Autoestima) nos revela una confiabilidad alta en todas ellas según los grupos comparados (Sin Abandono, Con abandono Moral y en Ambos grupos); ésta tiende a situarse por encima del alfa 0.70 (Tabla 2).

Según los resultados descritos, nos podrían permitir decir que los resultados obtenidos por los grupos comparados tanto en Cohesión, Adaptabilidad y Autoestima son bastante confiables, ya que se han encontrando confiabilidades bastante altas en cada uno y en ambos grupos. 
Tabla 2: Confiabilidad Alfa de Cronbach de las pruebas de Cohesión, adaptabilidad y autoestima según los grupos comparados

\begin{tabular}{|c|c|c|c|}
\hline Variables & $\begin{array}{c}\text { Sin abandono } \\
\text { moral }\end{array}$ & $\begin{array}{c}\text { Con abandono } \\
\text { moral }\end{array}$ & Ambos \\
\hline Cohesión & 0.71 & 0.88 & 0.87 \\
Adaptabilidad & 0.78 & 0.88 & 0.87 \\
Autoestima & 0.81 & 0.85 & 0.92 \\
\hline
\end{tabular}

\section{Estudio de la normalidad}

Los valores de Z de Kolmogorov-Smirnov para las tres pruebas (Cohesión, Adaptabilidad y Autoestima) no resultan significativos (los valores superan el 0.01); es decir, se acepta la hipótesis de normalidad en cada una de las tres pruebas según los grupos comparados. (Tabla 3).

\section{Tabla 3: Normalidad de las Pruebas de Cohesión, Adaptabilidad y Autoestima según los grupos comparados mediante la Prueba de Kolmogorov-Smirnov}

\begin{tabular}{|l|l|l|l|l|l|l|}
\hline & \multicolumn{2}{|c|}{$\begin{array}{c}\text { Sin abandono } \\
\text { moral }\end{array}$} & \multicolumn{2}{c|}{$\begin{array}{c}\text { Con abandono } \\
\text { moral }\end{array}$} & \multicolumn{2}{c|}{ Ambos } \\
\hline VARIABLES & Z & Sig. & $Z$ & Sig. & $Z$ & Sig. \\
\hline COHESIÓN & 1,01 & 0,26 & 1,02 & 0,25 & 1,01 & 0,26 \\
\hline ADAPTABILIDAD & 0,84 & 0,48 & 1,63 & 0,01 & 1,13 & 0,15 \\
\hline AUTOESTIMA & 0,91 & 0,38 & 1,36 & 0,05 & 1,22 & 0,10 \\
\hline
\end{tabular}

\section{Estudio de las diferencias entre puntuaciones medias}

Se observa en la Tabla 4 que existen diferencias significativas entre la cohesión, adaptabilidad, autoestima y los grupos comparados. Se aprecia que el grupo sin abandono moral se encuentran mejor situados en estos tres aspectos que el grupo abandono moral y prostitución. Así el grupo sin prostitución, en Cohesión obtiene una media de 33,56 mientras que el grupo con abandono moral y prostitución una media de 25,84. En Adaptabilidad y Autoestima las diferencias se dan en el mismo sentido, tal como se aprecia en el Grafico 3.

Estudio de la asociación entre los tipos de familia y los grupos de «sin abandono moral» y en «abandono moral»

En la Tabla 5 . se puede apreciar que existe asociación altamente significativa $(\mathrm{p}<0.01)$ entre los tipos de familias y el presentar o no prostitución. El tipo de familia Extrema se da en un mayor porcentaje en el grupo con abandono moral y prostitución con un 51,25\% frente a un $5 \%$ en el otro grupo. Finalmente, el, grupo con abandono moral hay sólo un $3,75 \%$ de evaluados dentro del Tipo de familia Balanceada frente a un 46,25 en el grupo con abandono moral y prostitución. 
Tabla 4: Diferencias de Medias de las Pruebas de Cohesión, Adaptabilidad y Autoestima según los grupos comparados mediante la Prueba t de Student.

\begin{tabular}{|c|c|c|c|c|c|c|}
\hline \multirow{3}{*}{ COHESION } & GRUPO & N & Media & DE & t & \multirow{2}{*}{ Sig. } \\
\cline { 2 - 7 } & Sin abandono moral & 80 & 33,6 & 5,7 & 6,07 & \multirow{2}{*}{$0.0 * *$} \\
& En abandono moral & 80 & 25,8 & 9,8 & & \multirow{2}{*}{$0.0 * *$} \\
\multirow{2}{*}{ ADAPTABILIDAD } & Sin abandono moral & 80 & 31,3 & 5,7 & 6,77 & \\
& En abandono moral & 80 & 22,5 & 102 & & \multirow{2}{*}{$0.0 * *$} \\
\hline \multirow{2}{*}{ AUTOESTIMA } & Sin abandono moral & 80 & 35,0 & 8,6 & 14,00 & \\
& En abandono moral & 80 & 16,3 & 8,3 & & \\
\end{tabular}

** Significancia $<0.01$

Grafico 2

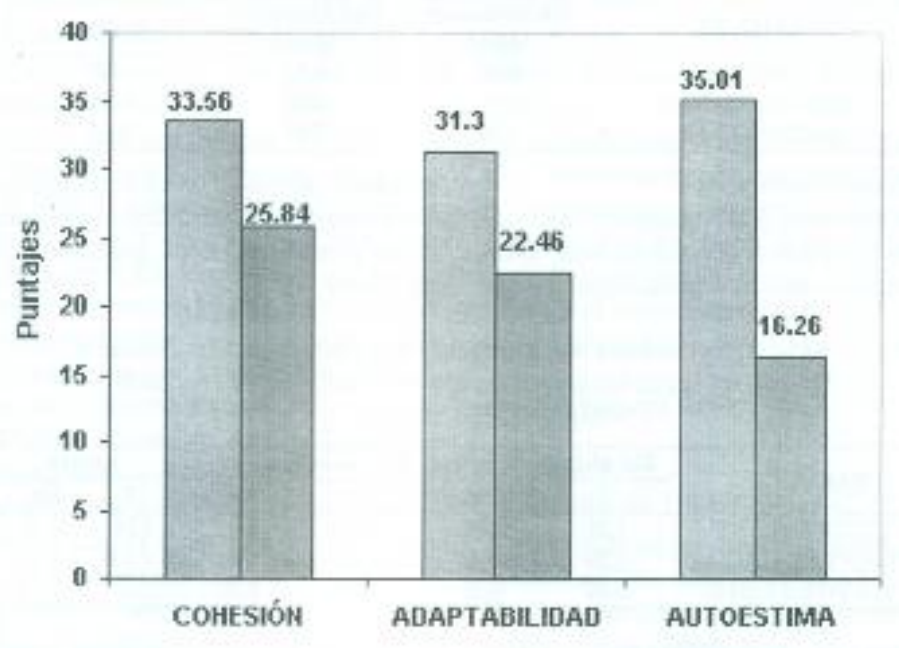

$\square$ Sin abandono moral $\square$ En abandono moral

Tabla 5: Asociación entre Tipo de Familia y los grupos comparados mediante la Prueba Chi cuadrado

\begin{tabular}{|c|c|l|l|l|}
\hline $\begin{array}{c}\text { TIPO DE } \\
\text { FAMILIA }\end{array}$ & $\begin{array}{r}\text { Sin } \\
\text { abandono } \\
\text { no }\end{array}$ & $\begin{array}{c}\text { En abandono } \\
\text { Moral }\end{array}$ & Ambos \\
\hline Extrema & Casos & 4 & 41 & 45 \\
& $\%$ & $5 \%$ & $51,3 \%$ & $28,1 \%$ \\
\cline { 2 - 5 } Rango medio & Casos & $39 \%$ & 36 & 75 \\
& $\%$ & $48,8 \%$ & $45 \%$ & $46,9 \%$ \\
\cline { 2 - 5 } Balanceada & Casos & $37 \%$ & 3 & 40 \\
& $\%$ & $46,3 \%$ & $3,8 \%$ & $25 \%$ \\
\hline
\end{tabular}

Ji cuadrado: $59,44 \mathrm{gl}=2$ Significancia $=0,00$ 


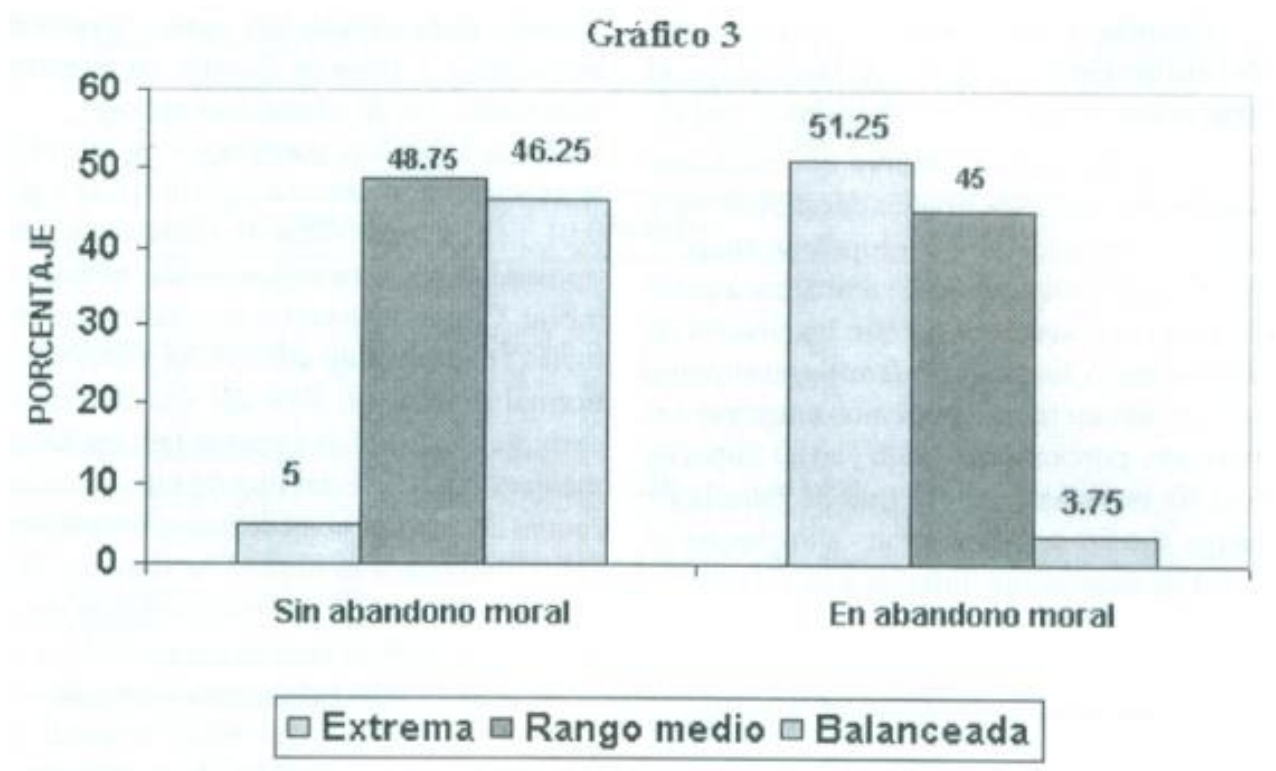

Asociación entre niveles de autoestima y los grupos de «sin abandono moral» y «con abandono moral»

En la Tabla 6 se aprecia que existe asociación altamente significativas $(p<0.01)$ entre los niveles de autoestima y los grupos con abandono moral y con abandono moral y prostitución. En el grupo con abandono moral y prostitución prevalece dentro del nivel de autoestima inferior a lo normal con un $62,5 \%$ frente a un $7,5 \%$ del grupo constraste el grupo sin abandono moral y prostitución alcanza un nivel ligeramente inferior a lo normal con un $25 \%$ frente al otro con $57,5 \%$.

Tabla 6: Asociación entre Niveles de Autoestima y los grupos comparados mediante la prueba de Chi Cuadrado

\begin{tabular}{|l|c|c|c|c|}
\hline \multicolumn{2}{|c|}{ Niveles de autoestima } & $\begin{array}{c}\text { Sin } \\
\text { abandono } \\
\text { moral }\end{array}$ & $\begin{array}{c}\text { Con } \\
\text { abandono } \\
\text { moral }\end{array}$ & Ambos \\
\hline $\begin{array}{l}\text { Muy inferior a lo } \\
\text { normal }\end{array}$ & $\begin{array}{c}\text { Casos } \\
\text { Inferior a lo normal }\end{array}$ & $\ldots$ & 10 & 10 \\
& Casos & $\ldots$ & 12.5 & 6.25 \\
\hline Ligeramente inferior a & Casos & 6.5 & 60 & 56 \\
lo normal & $\%$ & 57.5 & 20 & 35 \\
\hline Normal & Casos & 28 & 25 & 61.25 \\
\hline Ligeramente superior a & Casos & $\ldots$ & $\ldots$ & 28 \\
lo normal & $\%$ & $\ldots$ & $\ldots$ & 17.5 \\
\hline Superior a lo normal & Casos & $\ldots$ & $\ldots$ & $\ldots$ \\
& $\%$ & $\ldots$ & $\ldots$ & $\ldots$ \\
\hline
\end{tabular}

Ji cuadrado: $82,81 \mathrm{gl}=3$ Significancia $=0,00$ 
Estudio de la asociación entre niveles de autoestima y tipos de familia en el grupo con situación de abandono moral

En la Tabla 7 se observa que no existe asociación entre los niveles de autoestima y los tipos de familia en el grupo con abandono moral y prostitución. Si embargo, a pesar de no existir asociación entre los niveles de autoestima y los tipos de familia en el grupo con abandono moral, podemos notar que los mayores porcentajes tanto en el tipo de familia extrema como el tipo de familia de rango medio se encuentran situadas en el nivel de autoestima inferior a lo normal.

Estudio de la asociación entre niveles de autoestima y tipos de familia en el grupo «sin situación de abandono moral»

En la Tabla 8 se puede apreciar que existe asociación altamente significativas $(\mathrm{p}<0.01)$ entre los niveles de autoestima y los tipos de familia en el grupo sin abandono moral, Como se ve en los resultados porcentuales en el nivel de autoestima inferior a lo normal se sitúa un 50\% del tipo de familia extrema, un 7,69\% del tipo de familia Rango medio y un $2,70 \%$ del tipo de familia balanceada. Dentro del nivel de autoestima ligeramente inferior a lo normal se sitúa un $25 \%$ del Tipo de familia extrema, seguido de un $46,15 \%$ del tipo de familia rango medio y un $72,97 \%$ de familia balanceada dentro de esta categoría. Dentro del nivel normal de autoestima se sitúa un $25 \%$ de familia extrema, un $46,15 \%$ del tipo de familia de Rango medio, seguido de un $24,32 \%$ del tipo de familia balanceado.

Tabla 7. Asociación entre niveles de autoestima y tipos de familia en el grupo en situación de abandono moral mediante la prueba Chi cuadrado.

\begin{tabular}{|c|c|c|c|c|c|}
\hline \multirow{2}{*}{\multicolumn{2}{|c|}{ Niveles de autoestima }} & \multicolumn{3}{|c|}{ Tipo de familia } & \multirow{3}{*}{$\begin{array}{c}\text { Total } \\
10 \\
12.5\end{array}$} \\
\hline & & \multirow{2}{*}{$\begin{array}{c}\text { Extrema } \\
5 \\
12.2\end{array}$} & \multirow{2}{*}{$\begin{array}{c}\text { Rango } \\
\text { medio } \\
5 \\
13.9\end{array}$} & \multirow{2}{*}{$\begin{array}{c}\text { Balanceada } \\
\ldots \\
\ldots \\
\end{array}$} & \\
\hline $\begin{array}{c}\text { Muy inferior a lo } \\
\text { normal }\end{array}$ & $\begin{array}{c}\text { Casos } \\
\% \\
\end{array}$ & & & & \\
\hline Inferior a lo normal & $\begin{array}{c}\text { Casos } \\
\%\end{array}$ & $\begin{array}{c}30 \\
73.2 \\
\end{array}$ & $\begin{array}{c}19 \\
52.8 \\
\end{array}$ & $\begin{array}{c}1 \\
33.3 \\
\end{array}$ & $\begin{array}{c}50 \\
62.5 \\
\end{array}$ \\
\hline $\begin{array}{c}\text { Ligeramente } \\
\text { inferior a lo normal }\end{array}$ & $\begin{array}{c}\text { Casos } \\
\%\end{array}$ & $\begin{array}{c}6 \\
14.6\end{array}$ & $\begin{array}{c}18 \\
46.15 \\
\end{array}$ & $\begin{array}{c}2 \\
66.7\end{array}$ & $\begin{array}{l}20 \\
25\end{array}$ \\
\hline Normal & $\begin{array}{c}\text { Casos } \\
\%\end{array}$ & $\begin{array}{l}\cdots \\
\ldots\end{array}$ & $\begin{array}{l}\cdots \\
\ldots\end{array}$ & $\begin{array}{l}\cdots \\
\ldots\end{array}$ & $\begin{array}{l}\cdots \\
\ldots \\
\end{array}$ \\
\hline $\begin{array}{c}\text { Ligeramente } \\
\text { superior a lo } \\
\text { normal }\end{array}$ & $\begin{array}{c}\text { Casos } \\
\%\end{array}$ & $\begin{array}{l}\cdots \\
\cdots\end{array}$ & $\begin{array}{l}\cdots \\
\cdots\end{array}$ & $\begin{array}{l}\cdots \\
\cdots\end{array}$ & $\begin{array}{l}\cdots \\
\cdots\end{array}$ \\
\hline $\begin{array}{c}\text { Superior a lo } \\
\text { normal }\end{array}$ & $\begin{array}{c}\text { Casos } \\
\%\end{array}$ & $\begin{array}{l}\ldots \\
\ldots\end{array}$ & $\begin{array}{l}\ldots \\
\ldots\end{array}$ & $\begin{array}{l}\cdots \\
\ldots\end{array}$ & $\begin{array}{l}\cdots \\
\ldots\end{array}$ \\
\hline
\end{tabular}

Ji cuadrado: $6.98 \mathrm{gl}=3$ Significancia $=0.137$

Estudio de la asociación entre niveles de autoestima y tipos de familia en el grupo «sin situación de abandono moral»

En la Tabla 8 se puede apreciar que existe asociación altamente significativas ( $\mathrm{p}<$ 0.01 ) entre los niveles de autoestima y los tipos de familia en el grupo sin abandono moral, Como se ve en los resultados porcentuales en el nivel de autoestima inferior a lo 
normal se sitúa un 50\% del tipo de familia extrema, un 7,69\% del tipo de familia Rango medio y un 2,70\% del tipo de familia balanceada. Dentro del nivel de autoestima ligeramente inferior a lo normal se sitúa un $25 \%$ del Tipo de familia extrema, seguido de un $46,15 \%$ del tipo de familia rango medio y un $72,97 \%$ de familia balanceada dentro de esta categoría. Dentro del nivel normal de autoestima se sitúa un $25 \%$ de familia extrema, un $46,15 \%$ del tipo de familia de Rango medio, seguido de un $24,32 \%$ del tipo de familia balanceado.

Tabla 8: Asociación entre Niveles de Autoestima y Tipos de Familia en el grupo sin Situación de abandono moral mediante la Prueba Chi cuadrado

\begin{tabular}{|c|c|c|c|c|c|}
\hline \multirow{2}{*}{\multicolumn{2}{|c|}{ Niveles de autoestima }} & \multicolumn{3}{|c|}{ Tipo de familia } & \multirow{3}{*}{$\begin{array}{c}\text { Total } \\
\ldots \\
\ldots\end{array}$} \\
\hline & & \multirow{2}{*}{$\begin{array}{c}\text { extrema } \\
\ldots \\
\ldots \ldots\end{array}$} & \multirow{2}{*}{$\begin{array}{c}\begin{array}{c}\text { Rango } \\
\text { medio }\end{array} \\
\ldots \\
\ldots\end{array}$} & \multirow{2}{*}{$\begin{array}{c}\text { Balanceada } \\
\ldots \\
\ldots\end{array}$} & \\
\hline $\begin{array}{c}\text { Muy inferior a lo } \\
\text { normal }\end{array}$ & $\begin{array}{c}\text { Casos } \\
\%\end{array}$ & & & & \\
\hline Inferior a lo normal & $\begin{array}{c}\text { Casos } \\
\%\end{array}$ & $\begin{array}{c}2 \\
50\end{array}$ & $\begin{array}{c}3 \\
7.69\end{array}$ & $\begin{array}{c}1 \\
2.70\end{array}$ & $\begin{array}{c}6 \\
7.5\end{array}$ \\
\hline $\begin{array}{c}\text { Ligeramente } \\
\text { inferior a lo normal }\end{array}$ & $\begin{array}{c}\text { Casos } \\
\%\end{array}$ & $\begin{array}{c}1 \\
25\end{array}$ & $\begin{array}{c}18 \\
46.15\end{array}$ & $\begin{array}{c}27 \\
72.97\end{array}$ & $\begin{array}{c}46 \\
57.5\end{array}$ \\
\hline Normal & $\begin{array}{c}\text { Casos } \\
\%\end{array}$ & $\begin{array}{c}1 \\
25 \\
\end{array}$ & $\begin{array}{c}18 \\
46.15 \\
\end{array}$ & $\begin{array}{c}9 \\
24.32 \\
\end{array}$ & $\begin{array}{l}28 \\
25 \\
\end{array}$ \\
\hline $\begin{array}{l}\text { Ligeramente } \\
\text { superior a lo } \\
\text { normal }\end{array}$ & $\begin{array}{c}\text { Casos } \\
\%\end{array}$ & $\begin{array}{l}\cdots \\
\cdots\end{array}$ & $\begin{array}{l}\cdots \\
\cdots\end{array}$ & $\begin{array}{l}\cdots \\
\cdots\end{array}$ & $\begin{array}{l}\cdots \\
\cdots\end{array}$ \\
\hline $\begin{array}{c}\text { Superior a lo } \\
\text { normal }\end{array}$ & $\begin{array}{c}\text { Casos } \\
\%\end{array}$ & $\begin{array}{l}\cdots \\
\ldots\end{array}$ & $\begin{array}{l}\cdots \\
\ldots\end{array}$ & $\begin{array}{l}\cdots \\
\cdots\end{array}$ & $\begin{array}{l}\cdots \\
\cdots\end{array}$ \\
\hline
\end{tabular}

Ji cuadrado: $16,63 \mathrm{gl}=3$ Significancia $=0,002$

\section{DISCUSIÓN}

Los resultados obtenidos permiten inferir que el grupo sin abandono moral presenta una relación familiar más adecuada tanto en cohesión y adaptabilidad como en autoestima, es decir, son menores que experimentan un mayor vínculo emocional entre los miembros de la familia y poseen mayor habilidad en el sistema familiar para cambiar la estructura de poder, roles y reglas en las relaciones donde se requiere cambios.

Con respecto a la autoestima Coopersmith (984), señala que una adecuada autoestima es más probable que provengan de familias bien estructuradas, con límites y establecidos, tengan mayor confianza en sí mismo, sean más conscientes de sus propias opiniones y estén más dispuestos a expresar sus propias convicciones cuando enfrentan la oposición.

Por el contrario, las personas que son inseguras en relación a sus habilidades tienden a ser más cautelosas y no desean entrar en controversia o exponerse al criticismo. Coopersmith hipotetiza que los niños de baja autoestima son muy críticos de sí mismos y por tanto esperan el ser criticados por los demás, por lo que aceptan opiniones negativas acerca de ellos como algo natural.

Así en este estudio se observa que los menores en situación de abandono moral, es 
decir, menores que provienen de familias con una estructura no adecuada de funcionamiento, donde las reglas, los límites no son claros ni definidos, presentan una autoestima muy bajo de lo normal comparando con otro grupo que no se encuentra en situación de abandono moral o prostitución.

Además, sobre la base de estos resultados es factible opinar el grupo sin prostitución, habría una mayor adecuada interacción familiar expresada en la conexión y la adaptabilidad, así como una adecuada autoestima en comparación con el grupo con abandono moral y prostitución.

Como se sabe el sistema familiar juega un papel fundamental en la vida del individuo. Indudablemente, la familia es el primer marco de referencia en que se inicia la socialización y, por lo tanto, la personalidad del sujeto. La posición del individuo en su sistema familiar y la relación entre sus miembros tiene una gran influencia.

Si la estructura familiar no es saludable ejercerá una influencia negativa y nociva, caracterizada por el temor y el desarrollo atípico. Lo que los hijos aprenden de los padres dependen en parte del tipo de personas que estos sean. Esto significa que la familia se constituye en la principal fuente de conocimiento, valores, actitudes, roles y hábitos que se transmiten de una generación a otra.

En este estudio se ha podido apreciar que en los menores en situación de abandono moral y prostitución, prevalece dentro del tipo de familia Extrema. Según Olson (1984) estas familias son extremas tanto en las dimensiones de cohesión como adaptabilidad, es decir, tienen una tendencia a ser rígida con liderazgo autoritario y una disciplina estricta, y en la que rara vez se combina los roles y son familias que demuestran poca cercanía emocional entre sus miembros o una extrema cercanía emocional con alta dependencia y lealtad.

También podrá manifestar una tendencia caótica como falta de liderazgo y demasiado cambio entre el sistema familiar. Esta acentuada diferencia que hay entre la situación de abandono moral y las características que guarda una familia del rango Extrema, hace reflexionar una vez más sobre la importancia que esta encierra en relación al desarrollo y al actuar de sus miembros.

En referencia a los niveles de autoestima el grupo sin prostitución se encuentra en la mayoría de casos en los niveles ligeramente inferior a lo normal y en nivel normal en cambio $\mathrm{n}$ el grupo abandono moral y prostitución prevalece en los niveles muy inferior a lo normal, inferior a lo normal y ligeramente inferior a lo normal.

Todo lo expresado concuerda con un estudio realizado por UNICEF, (1994), que sostiene que las probabilidades de que el menor sea víctima de explotación sexual aumenta en la medida en que la familia tenga una imagen desvalorizada del menor y cree que se trata de una mercancía con la que se puede comerciar. Los daños experimentados por la menor víctima de esta epidemia como una pobre autoestima es usualmente severa como para que ellos se conviertan en potenciales suicidas.

En los resultados encontrados en el grupo de menores Sin situación de abandono moral podemos ver que existe relación altamente significativa entre los niveles de autoestima (inferior a lo normal, ligeramente inferior a lo normal y normal) y los tipos de familia (extrema, medio y balanceada). Esto indica que los menores que los menores que no se involucran en la prostitución poseen niveles de autoestima ligeramente inferior a lo normal y que corresponden a los tipos de familias del rango medio y balanceado.

Además se ha encontrado que la familia y la autoestima cumplen un papel relevante relacionado con la explotación de menores. La relación entre la percepción del tipo de familia y los niveles de autoestima que tienen los menores en situación de abandono, aunque no significativas, también se observan que pueden ser factores importantes en la 
predisposición a la explotación sexual de menores.

Evidentemente el fenómeno de la prostitución infantil como problema nacional debe ser enfocado desde una perspectiva multidisciplinaria, siendo urgente el promover el desarrollo de políticas sociales y económicas destinadas a promocionar los derechos de los niños.

Promover la creación de redes de hogares de protección y centros juveniles que no sólo se limite a la parte formativa, sino que también se promueva la creación de centros laborales que aseguren los ingresos necesarios a los menores para satisfacer sus necesidades básicas.

Incluir en el currículum educativo programas de educación sexual, educación en derecho con enfoque de género, así como programas de prevención contra el abuso sexual. Muchos programas de prevención muestran resultados positivos cuando se inician en la etapa preescolar y primaria mediante el aprendizaje de habilidades dirigidas a resistir al abuso sexual y a evitar la autoculpabilización.

Promover la cooperación internacional, fortaleciendo y reforzando las leyes para generar el bienestar de la infancia y proteger a la niñez contra el abuso. Estas leyes deben ponerse en práctica por medio de sanciones y penas severas contra los explotadores sexuales.

\section{REFERENCIAS BIBLIOGRÁFICAS}

Achancaray, B.; María I. \& Bazán, A. (1999). Aplicación de la Guía metodológica para favorecer el desarrollo de las habilidades sociales de asertividad, auto respeto, Autodisciplina y Complementariedad del Área de Personal Social en niños de El Agustino. Tesis, ISP Monterrico.

Bandura, A. (1987). Pensamiento y acción. Barcelona: Martínez Roca.

Cárdenas, B. (1989). Autoestima en niños urbano-marginales y su relación con el nivel de soporte social brindado por padres y pares. Tesis, USMP.

Castillo, C. (1999). Autoestima y su relación con la percepción de actitudes de los progenitores en niños de condición socio-económica media y baja. Tesis. UNIFE.

Coopersmith, S. (1967). The antecedents of self-esteem. A factor analytic study educational and Psychological Measurement. San Francisco.

Coopersmith, S. (1959). A method for determining types of self-esteem. Journal of Abnormal and Social Psychology, 59; 87-94.

Olson, D.; Portner, J. y Lavee. (1985). Faces Il. Family adaptability \& cohesion evaluation scale.

Olson, D. H., Killorin, E. (1984). The chaotic flipper in treatment. Kaufman, Power to change: Alcoholism. Gardner Press, Inc

Olson, D. H., \& Craddock, A. E. (1981).

Circumplex model ofmarital and family systems. Application to Australian families. Australian Journal of Sex, Marriage and Family, 1, 53- 69.

OMS/OPS (1999). Sociedad, violencia y salud. Memorias de la Conferencia Interamericana sobre violencia y salud, Ed. OPS, Washington.

ONG Acción por los Niños (1998). En: Reas de nocturnidad. Por O' Brien, P. Revista Somos del diario El Comercio, $\mathrm{N}^{\circ}$ 715, 19 de agosto, pp. 14-19. 
ONG Acción por los Niños (2000). En:

Prostitución infantil avanza en Lima. Por Cairo, P. Diario El Comercio, 20 de julio, sección B, p. 14.

Reusche, R. M. (1998). El funcionamiento familiar de adolescente con problema de conducta escolar, según la escala para evaluar la adaptación y cohesión de la familia, FACES IIl de Olson. Tesis para optar el grado de Doctora en Psicología, UNIFE. Lima, Perú.

Reusche, R. M. (1995). Estructura y funcionamiento familiar en un grupo de estudiantes de secundaria de nivel socioeconómico medio con alto y bajo rendimiento escolar. Revista de psicología, Universidad Femenina del Sagrado Corazón, N .3, pp. 163-190.

Reusche, R. M. (1994). El Modelo Circumplejo de funcionamiento familiar en la estructura familiar de adolescentes de nivel socioeconómico medio. Un estudio exploratorio en residentes. Lima: Tesis UNIFÉ

Save the Children (2002). Prostitución de niñas, niños y adolescentes. Documentos de Trabajo.

UNICEF (2001). Aprovecharse del abuso. Una investigación sobre la explotación sexual de nuestros niños y niñas. Nueva York.

UNICEF (2001). Explotación sexual y comercial de la infancia. Nueva York.

UNICEF, (1998). Segundo Congreso Mundial Contra la Explotación Sexual Comercial de los Niños. EstocolmoSuecia.

UNICEF, (1998). Estado de la niñez, la adolescencia y la mujer en el Perú, Lima.

UNICEF, (199)). Manual sobre maltrato y abuso sexual de los niños. México.

UNICEF, (1994). Estado mundial de la infancia. Barcelona.

UNICEF, (1991). Lineamientos y recomendaciones para la investigación prostitución infantil. Bogotá.

UNICEF, (1991). Análisis de la situación de menores en circunstancias especialmente dificil.

UNICEF, (1991). Hacia el rescate de la menor afectada por la prostitución. Santafé de Bogotá. 